\title{
A comprehensive study of synthesis and applications of core/shell nanoparticles
}

\author{
Anju Tiwari ${ }^{1}$, Ankit Kumar Tripathi ${ }^{2}$, Prateek Khare ${ }^{3^{*}}$ \\ ${ }^{1,2,3 *}$ Department of Chemical Engineering, Madan Mohan Malaviya University of Technology, Gorakhpur, INDIA \\ ${ }^{*}$ Corresponding Author: e-mail: ptkkhare@gmail.com, Tel +91-
}

\begin{abstract}
When we consider matter at nanoscale, one of the most important aspects to be considered is that due to the small size of the particles, these have increased surface area to volume ratio. The large value of this ratio increases the dominance of the surface atoms of the nanoparticles in relation to those in its interior. A special category of materials at nanoscale level has gained popularity in the recent times due to their interesting properties and applications. When classified on the basis of structure, the types of core/shell particles can have a large variety. Each of this structural classification has its own importance, method of synthesis and application. The core/shell nanoparticles have some distinct features that is responsible for their importance. The properties of Core/shell nanoparticles are highly modified from that of their simple pure nanomaterials, thus they usually called highly functional materials. The introduction of a different crust layer over the core particle has many reasons, such as it helps in implementing surface modification, increases functionality, stability and dispersibility, control on the release of the core, lowering the consumption of precious materials, and so on. These nanostructures can have a number of combinations in close interaction, depending upon the selection of material used, which highly influences the end application. This paper has an overview of the present methods involved in synthesis and tunable factors responsible for their end applications. The development of metal/metal oxide core-shell nanoparticles has become popular nowadays due to their widespread use in catalysis and other environmental remedial applications.
\end{abstract}

Keywords: nanoscale, surface modifications, functionality, tunable, environmental remedial applications.

DOI: http://dx.doi.org/10.4314/ijest.v13i1.23S

Cite this article as:

Tiwari A., Tripathi A.K., Khare P. 2021. A comprehensive study of synthesis and applications of core/shell nanoparticles. International Journal of Engineering, Science and Technology, Vol. 13, No. 1, pp. 153-157. doi: 10.4314/ijest.v13i1.23S

Received: December 1, 2019; Accepted: February 5, 2021; Final acceptance in revised form: March 31, 2021

This paper was earlier presented at the International Conference on Energy, Environment \& Material Sciences (ICE2M), 1-3 December 2019 and substantially improved for this Special Issue. Guest Editor: Dr. Sri Niwas Singh, Professor (HAG), Department of Electrical Engineering, Indian Institute of Technology Kanpur, 208016 (U.P.) India, former Vice-Chancellor, Madan Mohan Malviya University of Technology Gorakhpur (April 2017 to July 2020).

\section{Introduction}

Core/shell nanoparticles are the materials having varying properties. It consists of a core whose surface is modified with the deposition an exterior layered material which decreases its reactivity and increases the dispersibility and substantiality of the modified particle. The core/shell nanoparticles can be of varying shapes and sizes. These properties also affect their functionalities and their applications. These modified nanostructures can be grouped on the basis of combination of organic and inorganic materials used in these synthesis. In organic core and organic shell nanoparticles the core as well as the shell are organic materials or polymers. It has huge application in the drug delivery systems, in the catalysis and so on. These have modified properties such 
as rigidity, toughness and are generally referred to as smart materials.In the case of organic core and inorganic shell, these nanostructures consists of polymers or different copolymers as cores and shells are inorganic materials such as silica, metals or metal oxides. The inorganic shells provide rigidity whereas the organic core is spongy in nature, so it introduces flexibility in the material. Therefore, this increases the strength of the particle and the resistance to oxidation. While the inorganic core and inorganic shells has excellent performance with enhanced magnetic properties, energy absorption. Inorganic core and organic shell nanoparticles find applications in the field of paints, pigments, cosmetics etc. A variety of shapes of core/shell nanoparticles are also possible such as spherical, hexagonal, multiple small cores coated by single shell, yolk shell or movable core within hollow shell material. Generally, two schemes are used to vary the shapes of the structures synthesized. The first methodology involves an even layering of the shell material over a hard or soft core of different shapes resulting in the preparation of the desired shaped particles. The other method involves the use of a suitable capping agent or some of the reagent to control the required dimension and growth. The coating efficiency is generally found to be depending upon the shape of the core. It is generally seen that uniform coating phenomenon is reduced if the core has a distorted shape from spherical.

The non-spherical nanostructures have larger surface area relative to spherical ones due to the introduction of sides and corners, thus they perform better as catalysts. A number of research works have been published highlighting some special properties and their applications based on their end use. It is assumed that the core shell nanoparticles will replace almost all the existing current technologies and will play acrucial role in the enhancement of future research.

This paper targets to emphasise on the present status in the synthesis and application aspects of core/shell nanoparticles. The parameters which influence the properties and end uses of these nanostructures have also been used. This study demonstrates the development of modified nanostructures with desired properties by varying the synthesis parameters. These have great potential, different research groups over the globe are making efforts to simplify the synthesis and find innovative and reliable means to produce core/shell nanostructures of different sizes and shapes.

\section{Synthesis}

Various experimental parameters like temperature, time, concentration is varied to produce a wide range of core/shell nanoparticles. The core/shell nanoparticles synthesized for a particular application is to be designed by controlling and optimizing these parameters along with the selection of the appropriate material of the core/shell and the required shape. Full control over the process parameters along with the nucleation and growth of the shell is required to get the desirable characteristics in the synthesized particles. Some general methods are employed in the synthesis this class of nanoparticles, here these will be discussed in sections based upon the material of core/ shell nanoparticles. On the basis of material properties of the core/shell nanoparticles, these are classified as: organic/organic core/shell nanoparticles, organic/inorganic core/shell nanoparticles, inorganic/inorganic core/shell nanoparticles.

The organic/organic core/shell nanoparticles are also called smart particles due to their extensive use in different fields. These have a polymer coating over a polymer core which results in increase in overall toughness of the particles, the glass transition temperature also changes. Generally, polymerization mechanisms (cross-linking, free-radical and copolymerization) are used in the synthesis of these particles. In some cases, inorganic materials mixed with the polymers are used for adhesion that improves the mechanical properties the particles obtained. As an instance, during the synthesis blending $\mathrm{Sb}_{2} \mathrm{O}_{3}$ in the core increases the toughness and mechanical strength of PVC in PMMA/PVC core/shell nanostructures. (Xie et al, 2004). It has been observed that the properties of the inorganic materials are also affected besides the polymer (Lu et al, 2006)

The organic/inorganic core/shell nanoparticles have a polymer at core coated with a metal oxide, metalchalcogenides or silica shell. They possess dual properties due the presence of both an organic and an inorganic material. Since the core is organic, so it is prepared in a similar way as that of organic/organic core/shell nanostructures. Generally, the deposition of inorganic crust over the core is done using hydrolysis or sol-gel method followed by reduction, coprecipitation or calcination.

The inorganic/organic class of core/shell nanoparticles has a metallic or metal oxide core with a polymeric crust. Theshell material is organic, which makes the core oxidation resistant and biocompatible.The core synthesis involves wet chemical reactions, reduction or hydrolysis, the shell deposition involves polycondensation and polymerization reactions. The nanoparticles prepared by wet chemical reactions are crystalline and have good magnetization, so these are widely used for synthesis of magnetic core nanostructures. It is difficult to control the size of the synthesized particles due to high tendency of aggregation. A single-layered polymer coat can prevent the agglomeration to some extent, and it can be easily removed as per requirement. The dispersibility of silica particles can also be enhanced using the polymer layer.

The inorganic/inorganic core/shell nanostructures are, generally, made up of metal, metal oxide or silica. The synthesis of this class involves processes like microwave treatment, precipitation and hydrothermal decomposition. The crystal lattice parameters and the chemical interaction between the interface of core/shell nanoparticles are taken in account for selecting the materials to be used. In cases of incompatibility of lattice between the constituents, an appropriate surfactant or ligand is used for the formation of the coat over core. The use of metal precursor salts increases the possibility of aggregation during the preparation of core. So, it is tried to be avoided by using any alternative process. If the use of metal salts as precursors is inevitable then a proper surfactant is selected which prevents the problem of agglomeration. The surfactant helps in modifying the interfacial energy between the incompatible constituents. 
For an instance, the synthesis of $\mathrm{ZnO}$ shell over noble metals is very challenging as $\mathrm{ZnO}$ is hexagonal and it grows differently along the $\mathrm{c}$ axis. Thus, this synthesis was very problematic on account of the lattice mismatch; besides this no surfactant or ligand could help. (Wang, et al,2010 )This was recently solved to some extent with the synthesis of $\mathrm{Ag} / \mathrm{ZnO}$ core/shell nanoparticles. These were synthesized for the first time by laser ablation technique, using a $248 \mathrm{~nm} \mathrm{KrF}$ excimer pulsed laser ablation in a solution. (Zhao et al, 2015) Other attempts of coating $\mathrm{ZnO}$ were also made, a comprehensive growth mechanism stating a general scheme was developed. (Sun et al, 2013) Polyvinylpyrrolidone (PVP) and 4-mercaptobenzoic acid were crucial in the synthesis of $\mathrm{Au} / \mathrm{ZnO}$ core/shell nanoparticles. The amphiphilic nature of PVP helps in the deposition of $\mathrm{ZnO}$ coating on metallic core which have hydrophobic ligands.

The synthesis of modified shapes like hollow shell or yolk/shell nanostructures are synthesized for special applications. A simple synthesis method to get hollow $\mathrm{Pd} / \mathrm{CeO}_{2}$ core/shell nanoparticles consisting of $\mathrm{Pd}$ cores with $\mathrm{CeO}_{2}$ hollow shells is discussed in (Zhang et al, 2015). A one-pot hydrothermal reaction was used to prepare a metal/ Al2O3yolk/ shell nanoparticles (Zhang et al, 2015). A metallic core was coated by carbon that was then used for nucleation for a layer of Al2O3. When the hydrothermal reaction was extended, the carbon shell diameter increased by $100 \mathrm{~nm}$. The shell thickness could be controlled by varying the salt concentration. It has been demonstrated that changing the magnetite shell thickness, the magnetic properties can be altered to obtain multi-functionality and new improvised properties (Ivanov et al, 2016).

Properties such as blocking temperature, magnetization are dependent on the particle size while the catalytic activity, selectivity, melting point, optical properties varies with the shape of the particle. The efficiency of the desired shape coating also depends upon the shape of the core materials involved in the synthesis and its symmetrical nature. The size of the shell can be controlled by optimizing the concentration and reaction time for the synthesis. The shape can also be monitored by controlling the thermodynamic and kinetic parameters. The shape, phase of the medium and size influences the characteristics of magnetic core/shell nanoparticles. Magnetic nanoparticles have a wide range of applications and mainly Iron (III) Oxide is mostly used as it hasmagnetic properties and chemical stability.

The kind of salt precursor or the capping reagent used and its concentration determines the shape of the synthesized core/shell nanoparticles. Later, it can be further characterized using different techniques. Thus, it has application in the process of the shapecontrolled synthesis of mainly magnetic core/shell nanoparticles. Certain core/shell nanoparticles of a definite shape can be synthesized using an appropriate salt, solvent and surfactant/capping reagent. With change in capping reagent concentration, the shape of the magnetic core/shell nanoparticles varies at lower concentration as in (Fatima et al, 2018).

Variation in shape changes the surface exposed and also alters the crystalline arrangements thus it allows varied applications. Moreover, the rate of formation of nanoparticles can be increased to a greater extent by adopting the shape variation techniques. The optimization of parameters to obtain $\mathrm{Fe}_{3} \mathrm{O}_{4}$ of cubical and tetrahedron shapes has been discussed in (Fatima et al, 2018).. It was cubic when $0.5 \mathrm{M} \mathrm{KOH}$ (capping agent) was used and on increasing the concentration to $1 \mathrm{M}$ octahedron shape was observed. This happened as increase in the hydroxyl concentration to $1 \mathrm{M}$ fastens the rate of formation of octahedron shape rather than cubic at the lower concentration. The octahedron shape was formed after achieving the cubical shape by growth at the cubical corners as in (Fatima et al, 2018). When the concentration of $\mathrm{KOH}$ was further increased, it led to unsymmetrical growth and distortion from octahedron shape. It has been found that crystal growth occurred in different planes and therefore different shapes were observed as in (Fatima et al, 2018). The anisotropy of the core/shell nanoparticles, which relates the regularity of arrangement in the particles, varies inversely with it. Considering the iron core, the magnetization values varies directly with the symmetry.

Table 1. Tuning parameters for controlling the morphology of core/shell nanoparticles

\begin{tabular}{|c|c|}
\hline PROPERTY & TUNING PARAMETERS \\
\hline Size & Reaction period \\
\hline Shape & $\begin{array}{c}\text { Type and concentration of } \\
\text { appropriate salt precursor, solvent } \\
\text { media and surfactant or capping } \\
\text { reagent. }\end{array}$ \\
\hline $\begin{array}{c}\text { Nature } \\
\text { (Amorphous) }\end{array}$ & Salt precursor concentration \\
\hline $\begin{array}{c}\text { Crust } \\
\text { thickness }\end{array}$ & $\begin{array}{l}\text { Salt precursor concentration } \\
\text { Reaction period }\end{array}$ \\
\hline
\end{tabular}

It was found that in the synthesis of octahedral $\mathrm{Au}$ core, incomplete coating was observed whereas spherical shapes were completely coated as in (Chaudhuri et al, 2012). Thus, the efficiency of formation of uniform shell decreases if the shape of the core is distorted from the sphere.

Magnetic iron core particles were synthesized and discussed in (Balakrishnan et al, 2009). The particles were synthesized by chemical reduction of $\mathrm{FeCl}_{2}$ in an aqueous medium with sodium borohydride. These were surfaced with amine-terminated poly(ethylene glycol). It was observed that the particles became more amorphous with the increase in reactant concentration.

Taking the case of the catalytic activity in the metallic nanoparticles, It was found as in (Narayanan et al, 2014) 
that it was more in the non-spherical shapes as compared with the spherical ones because of increase in the number of exposed surfaces and increase in the surface area. The losses in the yield mainly occur during the separation process.

\section{Applications}

Due the diversity in the size and shape of core and crust, type of materials used in synthesis, the core/shell nanoparticles are used extensively and have a broad stretch of applications. Classes of inorganic/inorganic core/shell nanoparticles are used on a larger scale for the photocatalytic purposes and are involved in the enhancement of the semiconductor efficacy, catalysis, optical properties etc. It can be further classified on the grounds of nature of the surface material containing mainly silica or any other inorganic material. Silica as a crust is involved in redox reactions, in reduction of the bulk conductivity, increasing stability etc. as in (Chaudhuri et al, 2012) thereby showing varied applications because of its inertness. The CdS/ZnS core/shell nanostructures have higher photocatalytic activity than pure $\mathrm{CdS}$ and $\mathrm{ZnS}$ as in (Reddy et al, 2017) for the degradation of methyl orange.

The core/shell nanoparticles with inorganic/inorganic composition, are also used in the enhancement of the electrochemical properties and sensor-based applications. Sensors made up of $\mathrm{Ag} / \mathrm{WO} 3$ nanostructures have enhanced performance and showed shorter recovery time when compared with $\mathrm{Ag}-\mathrm{WO}_{3}$ mixture, pure $\mathrm{WO}_{3}$ and $\mathrm{Ag}$, as in (Xu et al, 2014 ) thereby showing synergistic improvement in the sensor working phenomenon by altering the nature of the material due to increase in the sensitivity to pollution, thermally stable. It also shows adsorption properties and is widely used for removing harmful chemicals, pigments, colours and dyes. (Meng et al, 2016) used a combination of $\mathrm{SiO}_{2} / \mathrm{Co}(\mathrm{OH})_{2}$ core/shell nanoparticles for the adsorption of Rhodamine B (xanthene dye) that showed increase in the adsorption capacity of the dye pollutant from $0.14 \mathrm{mg} / \mathrm{mg}$ to $0.41 \mathrm{mg} / \mathrm{mg}$ with decrease in the dose of these particles from $7 \mathrm{mg}$ to $1 \mathrm{mg}$.

Inorganic core with organic shell nanoparticles are used in the pigments, drug delivery and absorption phenomenon; the increased oxidation stability of the core, electromagnetic shielding shows enhanced biocompatibility. It has also been used for water treatment as in (Sari et al, 2017). $\mathrm{Fe}_{3} \mathrm{O}_{4} /$ glucose core/shell nanoparticles and was capable of absorbing $93.78 \%$ of lead ions and thus promoted the removal of heavy metal ions from the wastewater as in (Sari et al, 2017).

If the nanostructures have organic core, these can be widely used for broad spectrum of applications. Core is organic and therefore a flexible nature of the particle is observed. It can handle certain amount of pressure and thereby used in the synthesis of a variety of construction and building materials, thermal and colloidal stability and provides oxidation resistance.

Organic/organic core/shell nanoparticles alter the rigidity and has wider applications in the pharmaceutical industry, biosensing, biomaterials etc. These can also be used as nano-reactors in the upcoming time. By change in the volume transition phenomenon within the thermosensitive shell the catalytic activity of the nanoparticles can also be regulated to a greater extent as in (Ballauff $e t$ $a l, 2007)$. It was found that volume transition within a thermosensitive network can be used as an on-off switch controller and with a tunable catalytic activity of nanoparticles of any order of magnitude more than one can occur.

\section{Discussion and Future Prospects and conclusion}

The stability and structural integrity of core/shell nanoparticles is intact even in aqueous media. These structures can be successfully applied for various applications including catalysis, biomedical and photocatalytic usage. The metal core and metaloxide shell nanoparticles have extraordinary scope of use in environmental remediation and bacterial disinfectants. The pharmaceutical industries have also been largely benefitted by use of core/shell nanoparticles. Further detailed study of these nanoparticles will also improve the present scenario in the field of wastewater treatment on a broader scale. Accounting to the excellent electronic and physiochemical properties, nanoscale structures of metals like $\mathrm{Au}, \mathrm{Pd}, \mathrm{Ag}$ and $\mathrm{Pt}$ are being explored extensively. Metal oxide shells possess good photocatalytic, magnetic, photon harvesting and electronic capability that makes them appropriate to be considered for developing excellent transistors, sensors, bioimaging and photovoltaic devices. The metal/semiconductor core/shell nanoparticles have a great potential in wastewater treatment as photocatalysts. The metal cores of noble metals like gold and silver with metal oxide shell have potential to be used in air purification. Moreover, these assemblies are very cost efficient. As nanotechnology is one of the burgeoning topics, this special category of nanostructures will play a significant role in the future, innovation and further strategies should be adopted to make it more energy efficient, reliable and economical.

\section{Acknowledgment}

Authors immensely acknowledge to the Department of Chemical Engineering, Madan Mohan Malaviya University of Technology, Gorakhpur, Uttar Pradesh, India. We would like to thank Assistant Professor Dr. Prateek Khare for his tremendous support and help. He gave his useful suggestions during the preparation of the manuscript and guided us to materialize it. We would also like to thank other faculties of the department for their guidance and consideration during our study. 


\section{References}

Balakrishnan S., Bonder M.J., Hadjipanayis G.C., 2009. Particle size effect on phase and magnetic properties of polymer-coated magnetic nanoparticles, Journal of Magnetism and Magnetic Materials, Vol. 321, pp. 117-122. https://doi.org/10.1016/j.jmmm.2008.08.055

Ballauff M., Lu Y., 2007. Smart nanoparticles: Preparation, characterisation and applications, Polymer, Vol. 48, No. 7, pp. 18151823. https://doi.org/10.1016/j.polymer.2007.02.004

Chaudhuri R.G., Paria S., 2012. Core/shell nanoparticles: Classes, properties, synthesis mechanisms, characterization, and applications, Chemical Reviews, Vol. 112, pp. 2373-2433. https://doi.org/10.1021/cr100449n

Fatima H., Lee D.-W., Yun H.J. and Kim K.-S. 2018. Shape-controlled synthesis of magnetic Fe3O4 nanoparticles with different iron precursors and capping agents, RSC Advances, Vol. 8, pp. 22917-22923. https://doi.org/10.1039/C8RA02909A

Garito F. A., Hsiao Y. L., Gao R., 2003. U.S. Patent, S20030174994 A1.

Ivanov Y. P., Alfadhel A., Alnassar M., Perez J. E., Vazquez M., Chuvilin A. and Kosel J.R., 2016. Tunable magnetic nanowires for biomedical and harsh environment applications, Sci. Rep., Vol. 6, 24189. doi.org\%2F10.1038\%2Fsrep24189

Lu Y., Mei Y., Ballauff M., Dreschler M., 2006. Thermosensitive core-shell particles as carrier systems for metallic nanoparticles, J. Phys. Chem. B., vol. 110, pp. 3930-3937, https://doi.org/10.1021/jp057149n.

Lu Y., Mei Y., Drechsler M., Ballauff M., 2006. Thermosensitive Core-Shell Particles as Carriers for Ag Nanoparticles: Modulating the Catalytic Activity by a Phase Transition in Networks, Angew. Chem. Int. Ed., Vol. 45, pp. 813-816, https://doi.org/10.1002/anie.200502731

Meng Y., 2015. Synthesis and adsorption property of $\mathrm{SiO}_{2} @ \mathrm{Co}(\mathrm{OH})_{2}$ Core-Shell Nanoparticles, Nanomaterials, Vol. 5, No. 2, pp. 554-564. https://doi.org/10.3390/nano5020554

Narayanan R., El-Sayed M.A., 2004. Shape-dependent catalytic activity of platinum nanoparticles in colloidal solution, Nano Letters, Vol. 4, No. 7, pp. 1343-1348. https://doi.org/10.1021/n10495256

Reddy C.V., Shima J., Cho M., 2017. Synthesis, structural, optical and photocatalytic properties of CdS/ZnS core/shell nanoparticles, Journal of Physics and Chemistry of Solids, Vol. 103, pp. 209-217. DOI: http://dx.doi.org/10.1016/j.jpcs.2016.12.011

Sari A.Y., Eko A.S., Candra K., Hasibuan D.P., Ginting M., Sebayang P., Simamora P., 2017. Synthesis, properties and application of glucose coated $\mathrm{Fe}_{3} \mathrm{O}_{4}$ nanoparticles prepared by co-precipitation method, IOP Conference series: Materials, Science and Engineering, Vol. 214, pp. 120-21, DOI:10.1088/1757-899X/214/1/012021

Sun H., He J., Wang J., Zhang S.-Y., Liu C., Sritharan T., Mhaisalkar S., Han M.-Y., Wang D. and Chen H., 2013. Investigating the multiple roles of polyvinylpyrrolidone for a general methodology of oxide encapsulation, Journal of American Chemical Society, Vol. 135, pp. 9099-9110. https://doi.org/10.1021/ja4035335

Xie X. L., Li R.K.Y., Liu Q.X., Mai Y.W., 2004. Structure- property relationships of in-situ PMMA modified nano-sized antimony trioxide filled poly(vinyl chloride) nanocomposites, Polymer, vol. 45, pp. 2793-2802, https://doi.org/10.1016/j.polymer.02.028

Xu L., Yin M.-L., Liu S., 2014. Agx@WO3 core-shell nanostructure for LSP enhanced chemical sensors, Scientific Reports, Vol. 4, 6745. https://doi.org/10.3390/nano5020554

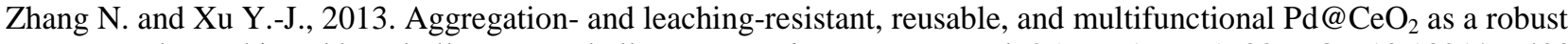
nanocatalyst achieved by a hollow core-shell strategy, Chem. Mater., Vol. 25, pp. 1979-1988. DOI: 10.1021/cm400750c

Zhang W., Lin X.-J., Sun Y.-G., Bin D.-S., Cao A.-M. and Wan L.-J., 2015. Controlled formation of metal@ $\mathrm{Al}_{2} \mathrm{O}_{3}$ yolk-shell nanostructures with improved thermal stability, ACS Appl. Mater. Interfaces, vol. 7, pp. 27031-27034. https://doi.org/10.1021/acsami.5b09791

Wang Z. L., 2010, Piezopotential gated nanowire devices: Piezotronics \& piezo-phototronics, Nano Today, Vol. 5, pp. 540-552. https://doi.org/10.1016/j.nantod.2010.10.008

Zhao Y., Li S., Zeng Y. and Jiang Y., 2015. Synthesis and properties of Ag/ZnO core/shell nanostructures prepared by excimer laser ablation in liquid, APL Mater., vol. 3, 086103. https://doi.org/10.1063/1.4928287

\section{Biographical notes}

Anju Tiwari received her B. Tech. in Chemical Engineering from Madan Mohan Malaviya University of Technology Gorakhpur, India in 2020 and is currently working as Junior Research Fellow at IIT Bombay.

Ankit Kumar Tripathi received his B. Tech. in Chemical Engineering from Madan Mohan Malaviya University of Technology Gorakhpur, India in 2020 and is currently working as Project Associate Fellow at CSIR-Institute of Minerals and Materials Technology, Bhubaneswar, Odisha.

Dr. Prateek Khare is an Assistant Professor in the Department of Chemical Engineering, Mohan Malaviya University of Technology Gorakhpur, India. He received M.tech. from National Institute of Technology Rourkela, India and PhD from IIT Kanpur. His current area of research includes Photocatalyst degradation, Adsorption, New and Renewable Energy (Microbial Fuel Cell), Advance Separation Process (Electrochemical Reduction). 